\title{
O EMBRIÃO DE THYRINTEINA ARNOBIA (LEPIDOPTERA: GEOMETRIDAE) PODE PRODUZIR PROTEÇÃO AO PARASITISMO DE ESPÉCIES DE TRICHOGRAMMA?
}

\section{CAN THE THYRINTEINA ARNOBIA (LEPIDOPTERA: GEOMETRIDAE) EMBRYO PROTECT THE TRICHOGRAMMA SPP. PARASITISM?}

\author{
Ulysses Rodrigues Vianna ${ }^{1}$; Nathália Suemi Saito ${ }^{2}$; Anderson Mathias Holtz ${ }^{2 *}$ \\ Dirceu Pratissoli ${ }^{2}$; Ricardo Antonio Polanczyk ${ }^{2}$
}

\begin{abstract}
RESUMO
Lepidópteros desfolhadores causam danos a plantios de Eucalyptus. Uma alternativa de controle é o uso de Trichogramma, que embora tenha sido relatado parasitando várias espécies em eucalipto, não se encontra casos de parasitismo em T. arnobia. O objetivo deste trabalho foi avaliar o parasitismo de Trichogramma pratissolii Querino \& Zucchi (Hymenoptera: Trichogrammatidae) em ovos inviáveis de T. arnobia. Ovos inviáveis de T. arnobia foram colados em retângulos de cartolina azul celeste, e expostos ao parasitismo de T. pratissolii por 24 horas. Após esse período observou-se mortalidade $100 \%$ dos indivíduos, sendo que os ovos não apresentaram parasitismo.
\end{abstract}

Palavras chave: Lepidópteros-desfolhadores, eucalipto, Trichogramma, parasitóide, controle biológico.

\begin{abstract}
Defoliator caterpillar cause damages in Eucalyptus plantations. A control alternative is the use of Trichogramma which, even though has been found parasitizing some species in Eucaliptus, does not meet cases of T. arnobia parasitism. The objective of this research was to evaluate the parasitism of Trichogramma pratissolii Querino \& Zucchi (Hymenoptera: Trichogrammatidae) on unviable T. arnobia eggs. Unviable T. arnobia eggs were glued on light blue cardboard rectangles, and exposed to the parasitism of T. pratissolii for 24 hours. After this period there was observed 100\% mortality of the individuals, though the glued eggs did not present parasitism.
\end{abstract}

Key-words: Defoliator caterpillar, Eucalyptus, Trichogramma, parasitoid, biological control.

Lepidópteros desfolhadores são insetos que podem causar danos a eucaliptocultura. Nesse grupo, estão incluídas pragas como Thyrinteina arnobia (Stoll) (Lepidoptera: Geometridae), considerada a principal praga em plantios de eucalipto no Brasil (Zanuncio et al., 1995; Santos et al. 1996).

Os surtos dessa praga em plantios de eucalipto têm sido controlados principalmente, utilizando-se inseticidas, que embora eficientes, podem causar a morte de inimigos naturais e ser responsáveis por impactos ambientais indesejáveis (Oliveira, 2001).
Dessa forma, têm-se buscado alternativas para o controle dessas pragas no setor florestal (Zanuncio et al., 1995).

Espécies do gênero Trichogramma (Hymenoptera: Trichogrammatidade) são inimigos naturais com ampla distribuição global, sendo altamente especializadas, eficientes e importantes para o manejo integrado de pragas de diversas culturas (Oliveira $e t$ al., 2000; Pratissoli et al., 2004, 2005). No Brasil, várias espécies de Trichogramma são importantes no controle de pragas agrícolas e florestais sendo

1 Departamento de Biologia Animal, Universidade Federal de Viçosa, 36571-000, Viçosa, Estado de Minas Gerais, Brasil. e-mail: ulyssesvianna@insecta.ufv.br.

2 Universidade Federal do Espírito Santo, Dep. de Fitotecnia, Centro de Ciências Agrárias, Caixa Postal 16, CEP 29.500-000, Alegre, Estado do Espírito Santo, Brasil. E-mail: nssaito@gmail.com, aholtz@insecta.ufv.br, dirceu@npd.ufes.br, Ricardo@ cca.ufes.br.

* Autor para correspondência.

Fecha de Recepción: 03 Febrero 2006

Fecha de Aceptación: 30 Mayo 2006 
relatadas parasitando muitas espécies de Lepidoptera em plantios de eucalipto (Oliveira et al., 2000).

Embora Trichogramma tenha sido relatado parasitando várias espécies de Lepidópteros-praga em eucalipto, não se encontra na literatura qualquer caso de parasitismo ou uso de parasitóides em programas de controle de T. arnobia. Isto devido à existência de um mecanismo de defesa presente nos ovos dessa espécie que provoca morte de $100 \%$ dos parasitóides em 24 horas de contato (Consoli et al., 1999; Oliveira, 2001).

Com o intuito de ampliar os conhecimentos sobre os mecanismos de defesa de T. arnobia visando à utilização de parasitóides do gênero Trichogramma em programas de controle biológico desta praga, o objetivo deste trabalho foi avaliar o parasitismo de Trichogramma pratissolii Querino $\&$ Zucchi (Hymenoptera: Trichogrammatidae) em ovos inviáveis de T. arnobia.

A pesquisa foi realizada no Centro de Ciências Agrárias da Universidade Federal do Espírito Santo, Alegre, Estado do Espírito Santo, Brasil, onde se encontra estabelecida a criação de T. arnobia em sacos de organza fixados em plantas de goiaba. Foi utilizada a espécie $T$. pratissolii, coletada em plantio comercial de eucalipto. A técnica empregada na criação de Anagasta kuehniella (Zeller) (Lep.: Pyralidae) seguiu a metodologia desenvolvida por Parra (1997).

Para a manutenção e multiplicação dos parasitóides, utilizaram-se ovos de A. kuehniella colados em retângulos de cartolina azul celeste $(8,0 \times 2,0 \mathrm{~cm})$, por meio de goma arábica diluída em água a 10\%, e inviabilizados pela exposição à lâmpada germicida (Parra, 1997). As cartelas foram acondicionadas em tubos de vidro contendo uma gotícula de mel puro para alimentação dos parasitóides, e que após serem lacrados com filme de PVC foram mantidos em câmaras climatizadas a $25 \pm 1{ }^{\circ} \mathrm{C}$, umidade relativa de $70 \pm 10 \%$ e fotofase de 14 horas.

Pupas fêmeas de T. arnobia foram isoladas em tubos de PVC contendo em seu interior papel branco para a obtenção de ovos inviáveis. Foram realizadas 10 repetições, sendo cada uma constituída por 15 ovos inviáveis de T. arnobia colados em retângulos de cartolina azul celeste $(2,5 \times 0,5$ $\mathrm{cm}$ ), por meio de goma arábica diluída em água a $10 \%$, e expostos ao parasitismo de uma fêmea de T. pratissolii por 24 horas em tubos de Duran $(3,5 \times 0,5 \mathrm{~cm})$, contendo uma gotícula de mel e lacrados com filme de PVC mantidos em câmaras climatizadas a $25 \pm 1{ }^{\circ} \mathrm{C}$, umidade relativa de $70 \pm 10 \%$ e fotofase de 14 horas.

Após o período de 24 horas de exposição dos parasitóides aos ovos inviáveis de T. arnobia observou-se mortalidade de $100 \%$ dos indivíduos, sendo que os ovos não apresentaram nascimento de descendentes do parasitóide.

$\mathrm{O}$ tratamento testemunha não foi utilizado para comparação dos resultados, pois a manutenção de T. pratissolii é realizada em ovos inviabilizados de A. kuehniella e não ocorre mortalidade dos indivíduos adultos.

Podemos constatar que a mortalidade observada dos adultos de $T$. pratissolii foi devido ao contato com os ovos inviáveis de T. arnobia, porém Oliveira (2001) observou mortalidade de $100 \%$ de fêmeas adultas de Trichogramma sobre ovos viáveis desse hospedeiro em menos de 24 horas. Sendo assim, outras hipóteses devem ser levantadas para explicar a mortalidade de espécies de Trichogramma sobre ovos de T. arnobia, já que essa mortalidade não é causada pelo embrião da mesma.

A mortalidade de T. pratissolii pode ser decorrente de compostos presentes nos ovos de T. arnobia, no entanto, esses compostos não são produzidos pelo embrião da mesma como um sistema de defesa. Uma hipótese é que essa mortalidade pode ser causada pela existência de uma camada mucosa sobre os ovos para auxiliar na adesão dos mesmos ao substrato e que também auxilia no reconhecimento e aceitação do parasitóide (Consoli et al., 1999) e, por um processo de adaptação e co-evolução $T$. arnobia pode utilizar-se dessa camada de adesão dos ovos como arma de defesa química contra parasitóides de ovos.

T. arnobia que antes se alimentava apenas de mirtáceas nativas mostrou uma grande adaptação para se alimentar de eucalipto (Holtz et al., 2003), principalmente, a partir de 1966 quando aumentou a área plantada com essa cultura no Brasil (Zanuncio et al., 1995; Santos et al., 2000), sendo que essas plantas apresentam em sua composição altas concentrações de compostos secundários (Ohmart \& Edwards, 1991) que podem estar interferindo, de alguma forma, no comportamento dessa espécie.

As plantas apresentam um sistema de defesa induzido, ativado quando herbívoros se alimentam (Agrawal, 2000). Entretanto, em resposta a esses mecanismos de defesa das plantas, os insetos evoluem de forma a suplantá-los (Maurício \& Rausher, 1997). Muitos insetos herbívoros conseguem se 
especializar em plantas com compostos secundários, e também em seqüestrar e utilizar esses compostos, se tornando impalatáveis e/ou igualmente tóxicos aos inimigos naturais (Agrawal et al., 1999), fator esse que pode estar causando a mortalidade dos parasitóides. Esses compostos podem ser utilizados então, na defesa contra os inimigos naturais, principalmente em suas fases mais vulneráveis como

\section{REFERÊNCIAS BIBLIOGRÁFICAS}

AGRAWAL, A. A. (2000). Specificity of induced resistance in wild radish: causes e consequences for two specialist and two generalist caterpillars. Oikos 89: 493-500.

AGRAWAL, A. A.; C. KOBAYASHI; J. S. THALER (1999). The influence of prey availability and induced host plant resistance on omnivory by western flower thrips. Ecology 80: 518-523.

CONSOLI, F. L.; E. W. KITAJIMA; J. R. P. PARRA (1999). Ultrastructure of the natural and factitious host eggs of Trichogramma galloi Zucchi and Trichogramma pretiosum Riley (Hymenoptera: Trichogrammatidae). International Journal of Insect Morphology \& Embryology 28: 211-231.

HOLTZ,A. M.; H. G. OLIVEIRA; A. PALLINI; M. VENZON; J. C. ZANUNCIO; C. L. OLIVEIRA; J. S. MARINHO; M. C. ROSADO (2003). Desempenho de Thyrinteina arnobia Stoll (Lepidoptera: Geometridae) em eucalipto e goiaba: o hospedeiro nativo não é um bom hospedeiro? Neotropical Entomology 32: 427-431.

MAURICIO, R.; M. D. RAUSHER (1997). Variation in the defense strategies of plants: are resistance and tolerance mutually exclusive? Ecology 78: 1301-1311.

NISHIDA R. (2002). Sequestration of defensive substances from plants by lepidoptera. Annual Review of Entomology 47: 57-92.

OHMART, C. P.; P. B. EDWARDS (1991). Insect herbivory on Eucalyptus. Annual Review of Entomology 36: 637-657.

OLIVEIRA, H.N.; J. C. ZANUNCIO; D. PRATISSOLI; I. CRUZ (2000). Parasitism rate and viability of Trichogramma maxacalii (Hym.: Trichogrammatidae) parasitoid of the Eucayiptus defoliator Euselasia apison (Lep.: Riodinidae), on eggs of Anagasta kuehniella (Lep.: Pyralidae). Forest Ecology and Management 130: 1-6. os ovos, permitindo a esses insetos uma maior proteção (Nishida, 2002).

Sendo assim, esses compostos utilizados como mecanismos de defesa devem ser mais estudados para que se possa traçar uma estratégia de controle dos surtos populacionais desta praga visando diminuir os custos de produção e o volume de inseticidas utilizado para esta finalidade.

OLIVEIRA, H. N. (2001). Potencial de uso de Trichogramma como agente de controle biológico de Lepidoptera desfolhadores de Eucalyptus e sua associação com percevejos predadores. Tese. Universidade Federal de Viçosa, Viçosa - MG, 78 pp.

PARRA, J. R. P. (1997). Técnicas de criação de Anagasta huehniella, hospedeiro alternativo para produção de Trichogramma, In: PARRA., J.R.P.; R.A. ZUCCHI (Eds.). Trichogramma e o controle biológico aplicado. Piracicaba: FEALQ, p. 121-150.

PRATISSOLI, D.; A. M. HOLTZ; J. R. GONÇALVES; R. C. OLIVEIRA; U. R. VIANNA (2004). Características biológicas de linhagens de Trichogramma pretiosum, criados em ovos de Sitotroga cerealella e Anagasta kuehniella. Horticultura Brasileira 22: 396-399.

PRATISSOLI, D.; U. R. VIANNA; H. B. ZAGO; P. L PASTORI (2005). Capacidade de dispersão de Trichogramma em tomateiro estaqueado. Pesquisa Agropecuária Brasileira 40: 613-616.

SANTOS, G. P.; J. C. ZANUNCIO; T. V. ZANUNCIO (1996). Pragas do eucalipto. Informe Agropecuário 9: 63-71.

SANTOS, G. P.; T. V. ZANUNCIO; J. C. ZANUNCIO (2000). Desenvolvimento de Thyrinteina arnobia Stoll (Lepidoptera: Geometridae) em folhas de Eucalyptus urophylla e Psidium guajava. Anais da Sociedade Entomológica do Brasil 29: 13-22.

ZANUNCIO, J. C.; J. B. TORRES; R. N. C. GUEDES; A. C. OLIVEIRA (1995). Tebufenozide, inseticida hormonal no controle da lagarta desfolhadora Thyrinteina arnobia Stoll (Lepidoptera: Geometridae). Anais da Sociedade Entomológica do Brasil 24: 599-604. 